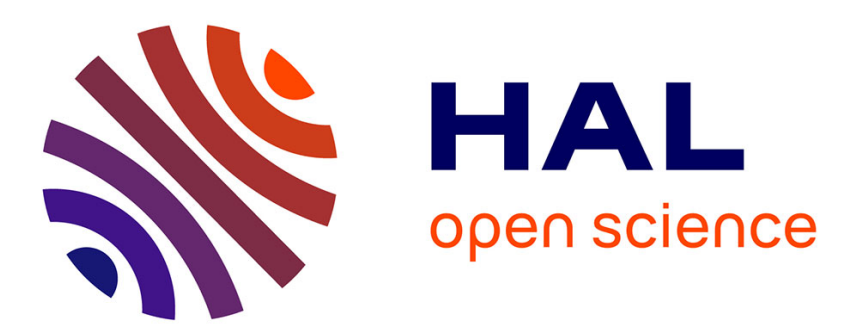

\title{
Three-Dimensional Modeling of Annular Cascade Trailing-Edge Noise
}

Michel Roger, Benjamin François, Michael Bauerheim

\section{To cite this version:}

Michel Roger, Benjamin François, Michael Bauerheim. Three-Dimensional Modeling of Annular Cascade Trailing-Edge Noise. 22nd AIAA/CEAS Aeroacoustics Conference, May 2016, Lyon, France. pp.1-18, 10.2514/6.2016-2949 . hal-02154844

\section{HAL Id: hal-02154844 \\ https://hal.science/hal-02154844}

Submitted on 13 Jun 2019

HAL is a multi-disciplinary open access archive for the deposit and dissemination of scientific research documents, whether they are published or not. The documents may come from teaching and research institutions in France or abroad, or from public or private research centers.
L'archive ouverte pluridisciplinaire HAL, est destinée au dépôt et à la diffusion de documents scientifiques de niveau recherche, publiés ou non, émanant des établissements d'enseignement et de recherche français ou étrangers, des laboratoires publics ou privés. 


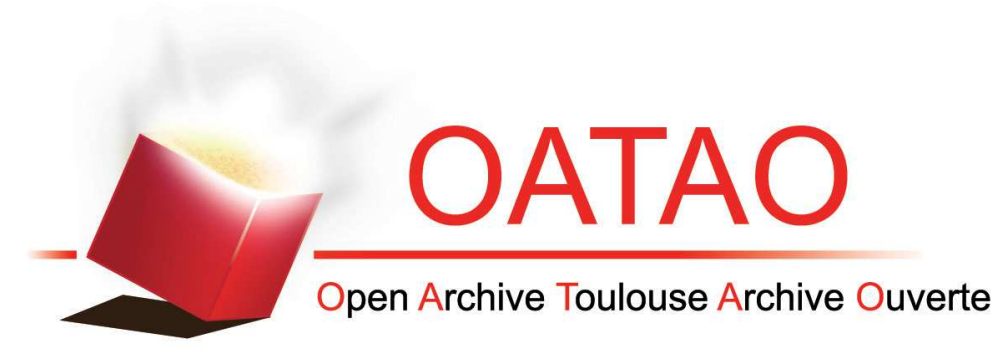

\section{Open Archive Toulouse Archive Ouverte (OATAO)}

OATAO is an open access repository that collects the work of some Toulouse researchers and makes it freely available over the web where possible.

This is an author's version published in: https://oatao.univ-toulouse.fr/20712

Official URL : http://doi.org/10.2514/6.2016-2949

\section{To cite this version :}

Roger, Michel and François, Benjamin and Bauerheim, Michael Three-Dimensional Modeling of Annular Cascade Trailing-Edge Noise. (2016) In: 22nd AIAA/CEAS Aeroacoustics Conference, 30 May 2016 - 1 June 2016 (France).

Any correspondence concerning this service should be sent to the repository administrator: tech-oatao@listes-diff.inp-toulouse.fr 


\title{
Three-Dimensional Modeling of Annular Cascade Trailing-Edge Noise
}

\author{
Michel Roger*, Benjamin François ${ }^{\dagger}$ and Michael Bauerheim ${ }^{\ddagger}$ \\ LMFA, Ecole Centrale de Lyon (Univ. Lyon), Ecully 69134, France
}

\begin{abstract}
The paper addresses various aspects of an analytical methodology for the modeling of the sound transmission through the outlet guide vanes of an axial-flow fan architecture, in view of predicting the trailing-edge noise with a proper account of the cascade effect. The first part extends a previous two-dimensional model by including the stagger and the curvature of the vanes. This is achieved by iteratively solving matching equations at the leadingedge and trailing-edge interfaces of the stator, with a multiple-scale analysis between two iterations, considering the inter-vane channels as bifurcated waveguides of slowly varying cross-section. The second part is aimed at extending a previous two-dimensional cascade trailing-edge noise model to an annular cascade described in cylindrical coordinates. For this the trailing-edge noise sources of a vane section are replaced by an equivalent lift dipole, the direct sound of which is expanded as a series of annular-duct modes. The scattering of each mode by the complete cascade is calculated by a three-dimensional mode-matching technique and the complete radiation of the trailing-edge source obtained by summing all modal contributions. The last part explains how the aforementioned two-dimensional model of curved-channel can be generalized in the three-dimensional annular geometry at the price of some approximations. Only preliminary results are given at each step, the paper being aimed at demonstrating the methodology but not yet at simulating a complete configuration. The objective is to formulate three-dimensional blade/vane row aeroacoustic problems without resorting to a strip-theory approach.
\end{abstract}

\section{Introduction}

The minimum arrangement addressed in the present work and considered as representative of many axial-flow fan architectures is made of a rotor and a downstream row of stationary outlet guide vanes (OGV) called stator. The role of the stator is to recover the swirl induced by the rotating blades of the rotor for an increased aerodynamic efficiency, so that the mean flow can be considered as ideally axial both upstream of the rotor and downstream of the stator. According to the acoustic analogy for subsonic Mach numbers, the aerodynamic noise of the rotor-stator stage is caused by the fluctuating lift forces on the blades and the vanes resulting from various aerodynamic interactions. These forces act as equivalent dipoles and produce either tonal noise or broadband noise depending on their periodic or random nature, respectively. In absence of additional distortions due to the installation the tonal noise originates from two mechanisms that are intrinsic to the design. On the one hand the mean velocity deficit in the wakes of the rotor blades generates tonal noise when impinging on the stator vanes. This is referred to as wake-impingement noise. On the other hand the potential field around the front part of the stator vanes contaminates the fluid farther upstream and induces lift fluctuations on the rotor blades. This is referred to as potential-interaction noise. The broadband noise is also caused by two main mechanisms associated with the turbulent part of the flow. Firstly wake turbulence generates random lift forces on the stator vanes and is responsible for the broadband part of wake-impingement noise. Secondly the turbulence developing over the blade and vane surfaces radiates sound as convected past the trailing edges. This is known as trailing-edge noise and is produced by both the rotor and the stator.

\footnotetext{
*Professor, AIAA Member

${ }^{\dagger}$ Post-doctorate research fellow

$\ddagger$ Post-doctorate research fellow
} 
According to this source breakdown both the rotor and the stator contribute to the acoustic signature of the stage. The acoustic waves can be expressed as sums of acoustic in-duct propagation modes. Each mode is transmitted through the duct or not depending on its cut-on or cut-off nature. Furthermore the upstream-propagating stator noise is scattered by the rotor, giving rise to reflected waves and transmitted waves that will be radiated outide the duct through the inlet. In the same way the downstream-propagating rotor noise is scattered by the stator, producing transmitted waves in the exhaust and upstream-traveling reflected waves. Both the generation and the transmission mechanisms must be clearly understood and captured in any sound prediction strategy, for the sake of designing low-noise architectures.

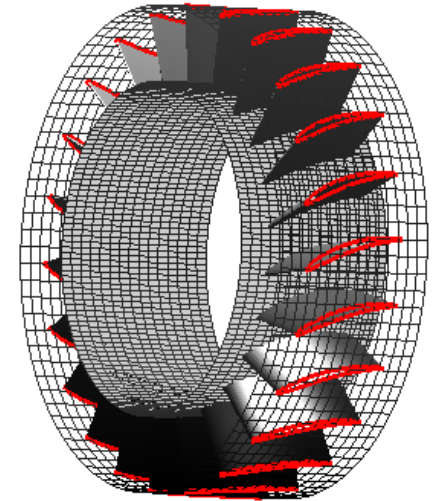

(a)

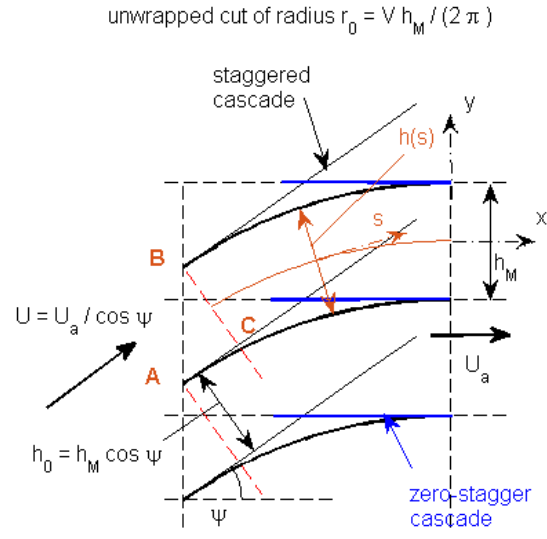

(b)

Figure 1: (a): typical axial-flow fan OGV. (b): unwrapped representation of a cylindrical cut.

The geometrical complexity of the stage makes the prediction task challenging, whatever the selected approach could be: numerical techniques are often limited by meshing/resolution issues or high computational costs, whereas analytical techniques can be limited by the necessary approximations. The advantages of the latter are their low cost and short computational times, allowing for parametric studies and optimization algorithms. This is why they remain attractive. The present work is therefore aimed at pushing the limits of analytical modeling by introducing more realistic design features than usually done in previous studies. One of the key issues when trying to derive response functions of blade or vane rows to acoustic or vortical excitations is to properly reproduce the cascade effect, defined as the effect of adjacent blades or vanes on the response of one of them. In a high-solidity case and for a large overlap of adjacent blades (vanes) the cascade effect is presumably important. Furthermore it also depends on the camber of the blades (vanes). In typical axial-flow architectures both the overlap and the curvature are more pronounced for the stator. This is why the present study concentrates on the response of a row of curved outlet guide vanes. The emphasis is on the transmission of acoustic waves but the last part explains how the approach can be used to formulate trailing-edge noise generation.

The investigated configuration is depicted in Fig. 1-a. The outlet guide vanes are assumed with zero twist and sweep. They are moderately cambered and staggered at leading edge and nearly parallel to the axis at trailing edge. As a result they have a large overlap and can be viewed as an array of periodic bifurcated waveguides. Except in the case of large hub-to-tip ratios for which the acoustic field is nearly homogeneous in the radial direction, the inter-vane channels are three-dimensional ducts of varying cross-section that must be described as such. For simplicity the OGV can be described in a two-dimensional unwrapped representation, as illustrated in Fig. 1-b for a cylindrical cut at an arbitrary radius $r_{0}$. The shortcoming of this simplification is that the effect of radial reflections on the duct walls are ignored. Moreover the unwrapped description in a Cartesian system of coordinates does not account for the azimuthal aperture of an inter-vane channel, say $2 \pi / V$ if $V$ is the number of vanes. Artificial multiple reflections between parallel walls take place in this representation. Yet it is retained in many reported studies for its ability to produce closed-form expressions of the sound field and/or of the dipole strengths. Typically the investigated annular cascade is cut in a 
series of thin annuli that are unwrapped and treated separately. This view is referred to as the strip-theory approach. It enables including arbitrary stagger angle, sweep and lean in the formulation by just changing the cascade parameters in each strip. But some effects of adjacent strips on the response of a reference strip are discarded. Amongst many authors having addressed sound generation or transmission problems in blade rows based on this approach, Glegg's model ${ }^{5}$ making extensive use of the Wiener-Hopf technique can be retained as a basis for subsequent works. It has been for instance used by Hanson to model resonance effects on rotor-stator broadband noise, ${ }^{6}$ and later re-addressed and extended by Posson et al. ${ }^{12}$ An alternative approach is developed in the present work, based on a mode-matching technique that can be extended in a three-dimensional annular configuration, at the price of some approximations. One of the main objectives is to finally avoid resorting to a strip-theory approach when reproducing the cascade effect.

A first two-dimensional statement of the mode-matching procedure applied to a cascade of zero-stagger vanes is described in a recent paper by Bouley et al, ${ }^{1}$ for the sake of validation by comparing with the Wiener-Hopf technique or other methods in some published test cases. The two-dimensional reduction of the model also allows developing a hybrid solution accounting for the stagger angle and the curvature of the vanes, as proposed recently. ${ }^{16}$ This work is re-addressed and completed in section I, leading to a uniformly valid expression of the sound field. The three-dimensional extension of the same approach is addressed in section II. The case of an annular cascade of zero-stagger plates is considered first because it can be formulated exactly and because the mean flow can be assumed axial and uniform without ambiguity, referring to a companion paper. ${ }^{2}$ The first step of a three-dimensional formulation of cascade trailing-edge noise is developed, extending the definition of the equivalent edge-dipole introduced in a previous work. ${ }^{18}$ Accounting for the stagger angle and the curvature of the vanes is much more challenging. The basis for ongoing work aimed at solving this issue is also described.

\section{Two-Dimensional Formulation for Bent Inter-Vane Channels}

\section{I.A. Preliminaries}

As shown by Roger et $a l^{18}$ the trailing-edge noise sources of an isolated vane can be reproduced by introducing an equivalent lift dipole approached very close to the trailing edge from downstream. In a twodimensional formulation the direct field of this dipole is expanded in a set of oblique plane waves and the scattering by the periodic array of inter-vane channels is obtained by summing the diffracted fields of the plane waves. In the reference the diffracted field was computed using a two-dimensional mode-matching technique, describing the stator as an array of zero-stagger flat plates. More recently Roger \& François ${ }^{16}$ raised two major issues related to this simplification, with regard to the stagger angle and the curvature of the vanes recognized as key design parameters of a stator. The stagger angle defines the orientation of the equivalent acoustic dipoles according to the acoustic analogy, and the curvature ensures the swirl recovery. Assimilating the vanes to flat plates is ambiguous because the choice of the equivalent stagger angle is arbitrary, with two extreme values: for slightly-loaded vane conditions, the incoming mean flow should be prescribed parallel to the vanes at the leading edges, whereas the exit flow should be purely axial. Sound transmission calculations performed with both extreme values were shown to lead to substantially different results. Furthermore, because of the camber of the vanes, the inter-vane channels are diverging from inlet to outlet. This makes channel waves propagating upstream from the trailing edges possibly experience total reflection because of cut-on to cut-off transition. Both aspects were discussed by the authors, separately for the sake of pointing out key features of the transmission mechanisms. They are re-addressed and combined in this section.

The need to include camber effects in the analytical response of the stator has been recognized, for instance, by de Laborderie et al, ${ }^{17}$ who adaptated the aformentioned Wiener-Hopf technique by assuming different stagger angles in the mathematical expressions for terms corresponding to the leading edges and the trailing edges. But this again was achieved on the basis of flat plates, ignoring the continuous effect of curvature on the sound transmission through an inter-vane channel. A different approach is developed in the present work, based on a mode-matching technique. Two different statements of the mode matching are formulated for the trailing-edge interface and the leading-edge interface, and then coupled in an iterative procedure. The curvature is introduced between successive iterations by assimilating the inter-vane channels to ducts of varying cross-section and performing a multiple-scale analysis. In the paper the latter is based on Rienstra's and Ovenden's developments, ${ }^{14,19}$ which means that only the continuous cross-section variation of the diverging channels is accounted for and that the curvature is neglected. Once determined in the 
straightened representation of a channel, the acoustic field is simply projected back onto the true channel. This simplification could be avoided; it is retained here for the sake of illustrating the method without additional complexity. Its justification is given in section I.C. At the trailing-edge interface the vanes are parallel to the axis and can be assimilated to zero-stagger parallel plates. Matching equations are obtained in this case in a standard way by imposing the continuity of the acoustic pressure and axial velocity. Furthermore a Kutta condition is added to force the pressure jump to zero between both sides of a vane just upstream of the trailing edge. This is not further discussed here because already detailed in the references. ${ }^{1,18}$ The matching at inlet where adjacent vanes do not overlap because of the stagger at leading edge must be formulated differently. A first method based on the use of Green's theorem, inherited from the theory of electromagnetic waves, ${ }^{20}$ was implemented by the authors. ${ }^{16}$ This method forbids access to the part of the field included in the matching triangle of corner C in Fig. 1-b. Though this is not prejudicial to the determination of the field away from the triangle, a straightforward extension of the matching procedure to an oblique interface is proposed in the section I.B, leading to a uniformly valid field description.

\section{I.B. Mode-Matching at Inlet}

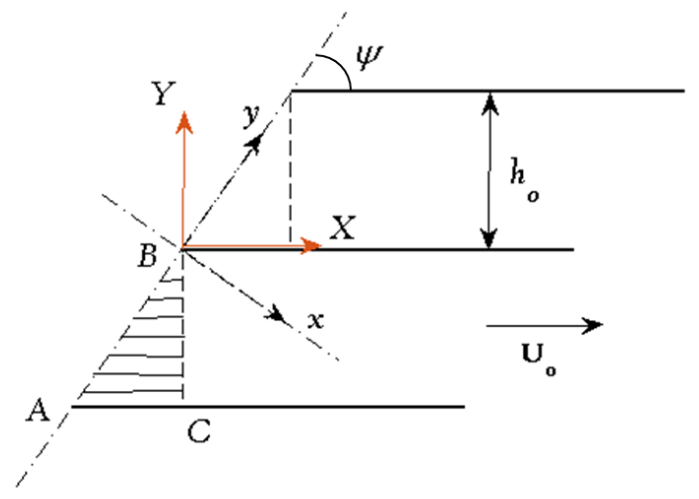

Figure 2: Reference frames for the staggered array of semi-infinite plates.

The zero-stagger interface is a simple configuration that can be solved by the standard mode-matching technique described by Mittra \& Lee ${ }^{11}$ for electromagnetic wave problems. Provided that changes of variables are performed to account for the presence of a uniform mean flow the formalism can be applied to aeroacoustic problems. If the interface corresponds to the trailing edges of a vane row the additional implementation of a Kutta condition is required, as described by Roger $e l$ all ${ }^{18}$ in a simplified model of trailing-edge noise for a zero-stagger cascade. This section describes the extension of the technique to staggered semi-infinite vanes in presence of a mean flow of speed $\mathbf{U}_{0}$ parallel to the vanes. The configuration is shown in Fig. 2; it corresponds to an interface inclined by an angle $\psi$, representative of the tangent to the mean camber-line at the leading-edge in Fig. 1-b. The flow is along the $X$-direction, oblique with respect to the matching interface of normal coordinate $x$. Even in this case, the acoustic pressure and velocity are continuous at the interface. They are related to the potential field $\phi$ as

$$
p^{\prime}=-\mathrm{i} \omega \rho_{0} \phi+\rho_{0} \mathbf{U}_{0} \cdot \nabla \phi \quad \text { and } \quad \mathbf{u}^{\prime}=\nabla \phi
$$

This linear relationship suggests that the continuity of $\phi$ and $\partial \phi / \partial X$ is also satisfied ${ }^{\mathrm{a}}$.

These continuity conditions are applied at the interface to match the incident wave of potential $\phi_{i}$ with an angle $\theta_{i}$ to the reflected and transmitted waves of potentials $\phi_{r}$ and $\phi_{t}$ in the channel, with

$$
\phi_{i}=\mathrm{e}^{\mathrm{i} \alpha_{i 0} y} \mathrm{e}^{\mathrm{i} K_{i 0} x}, \quad \phi_{r}=\sum_{s=-\infty}^{\infty} R_{s} \mathrm{e}^{\mathrm{i} \alpha_{r s} y} \mathrm{e}^{\mathrm{i} K_{r s} x}, \quad \phi_{t}=\sum_{p=0}^{\infty} T_{p} \cos \left(p \pi Y / h_{0}\right) \mathrm{e}^{\mathrm{i} K_{t p} X} .
$$

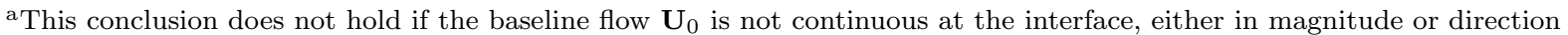


The wavenumbers depend on the axial Mach number $M_{0}$ or the coefficient $\beta=\sqrt{1-M_{0}^{2}}$, as

$$
\alpha_{i 0}=k \sin \theta_{i} /\left(1+M_{0} \sin \theta_{i}\right), \quad K_{i 0}^{+}=\frac{1}{\beta^{2}}\left(-M_{0} k+\sqrt{k^{2}-\beta^{2} \alpha_{i 0}^{2}}\right), \quad \alpha_{r s}=\alpha_{i 0}+2 \pi s / h_{0},
$$

where $h_{0}=h_{M} \cos \psi$ and

$$
K_{r s}^{-}=\frac{1}{\beta^{2}}\left(-M_{0} k-\sqrt{k^{2}-\beta^{2} \alpha_{r s}^{2}}\right), \quad K_{t p}^{+}=\frac{1}{\beta^{2}}\left(-M_{0} k+\sqrt{k^{2}-\beta^{2}\left(p \pi / h_{0}\right)^{2}}\right) .
$$

Prior to applying the continuity conditions, the transmitted wave $\phi_{t}(X, Y)$ and its gradient are written in the coordinates $(x, y)$. This allows achieving the mode matching at the interface $x=0$. Note that the boundary conditions corresponding to a hard wall at $Y=0$ and $Y=h_{0}$ are already included in the expression of the potential $\phi_{t}$.

For the sake of simplicity, only the continuity of the potential is detailed here; it reads

$$
\mathrm{e}^{\mathrm{i} \alpha_{i 0} y}+\sum_{s=-\infty}^{\infty} R_{s} \mathrm{e}^{\mathrm{i} \alpha_{r s} y}=\frac{1}{2} \sum_{p=0}^{\infty} T_{p}\left\{\mathrm{e}^{\mathrm{i}\left(p \pi / h_{0}+K_{t p}^{+} \sin \psi\right) y}+\mathrm{e}^{\mathrm{i}\left(-p \pi / h_{0}+K_{t p}^{+} \sin \psi\right) y}\right\} .
$$

Since all amplitudes $R_{s}$ and $T_{p}$ are unknown, a projection on the orthogonal shape functions of the reflected waves is performed to extract only the $\nu$-th reflected component $R_{s=\nu}$, expressed using all transmitted amplitudes $T_{p=0 \ldots \infty}$. In practice, Eq. (2) is multiplied by $\mathrm{e}^{-\mathrm{i} \alpha_{r \nu} y}$ and then integrated in the $y$-direction over the whole channel height, i.e., from 0 to $h_{0} / \cos \psi$. It yields three integrals that can be calculated analytically, leading to the projected equation

$$
h_{0}\left(\delta_{\nu, 0}+R_{\nu}\right)=\sum_{p=0}^{\infty} T_{p} \varphi_{p, \nu}(\psi)
$$

where

$$
\varphi_{p, \nu}(\psi)=\frac{-\mathrm{i} u_{p, \nu}(\psi)}{\left(p \pi / h_{0}\right)^{2}-u_{p, \nu}(\psi)^{2}}\left[1-(-1)^{p} \mathrm{e}^{\mathrm{i} h_{0} u_{p, \nu}(\psi)}\right] .
$$

This function generalizes the one obtained for non-staggered configurations, and depends on the stagger angle $\psi$ only through the wavenumber $u_{p, \nu}(\psi)=K_{t p}^{+} \sin \psi-\alpha_{r \nu}$. This is a formal advantage of using the natural extension of the mode-matching technique instead of Green's theorem; the latter leads to a different set of equations.

After some algebra, a similar equation is obtained using the same projection for the gradient of the potential as

$$
h_{0}\left(K_{i 0}^{+} \delta_{\nu, 0}+K_{r \nu}^{-} R_{\nu}\right)=\sum_{p=0}^{\infty} T_{p} \mathcal{K}_{p, \nu}^{+}(\psi) \varphi_{p, \nu}(\psi)
$$

where

$$
\mathcal{K}_{p, \nu}^{+}(\psi)=K_{t p}^{+} \cos \psi-\left(\frac{p \pi}{h_{0}}\right)^{2} \frac{\tan \psi}{u_{p, \nu}(\psi)} .
$$

Finally, to remove the unknown coefficient $R_{\nu}$, Eq. (4) is subtracted from Eq. (3) multiplied by $\mathcal{K}_{p, \nu}^{+}$, which yields the mode-matching equation. This equation coupling all coefficients $T_{p=0 . . \infty}$ can be truncated to $P$ modes, providing a finite linear system. In the matrix form, this system reads

$$
\left[M_{L E}\right][T]=\left[M_{L E}^{0}\right] \text { with }\left\{\begin{array}{l}
M_{L E}(\nu+1, p+1)=\varphi_{p, \nu}(\psi)\left[K_{r \nu}^{-}-\mathcal{K}_{p, \nu}^{+}(\psi)\right] \\
M_{L E}^{0}(\nu+1,1)=h_{0}\left[K_{r \nu}^{-}-K_{i 0}^{+}\right] \delta_{\nu, 0}
\end{array}\right.
$$

and the coefficients $R_{\nu}$ are obtained through

$$
R_{\nu}=\frac{1}{h_{0}} \sum_{p=0}^{P} T_{p} \varphi_{p, \nu}(\psi)-\delta_{\nu, 0}
$$

which ensures the pressure continuity. 


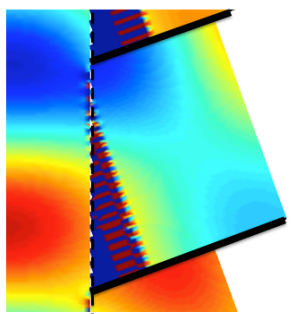

Green's theorem (GT)

(a)

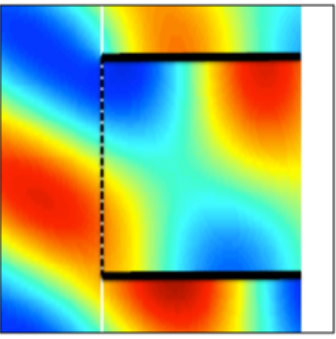

$\mathcal{E}=-0.02 \%$

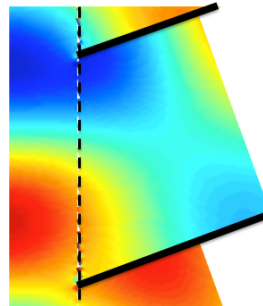

Mode matching (MM)

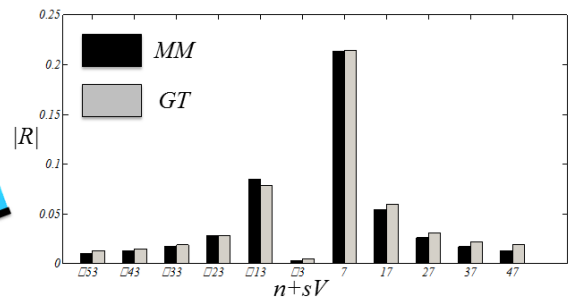

Reflection coefficients (b)

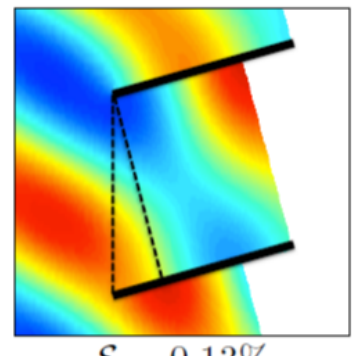

(c)
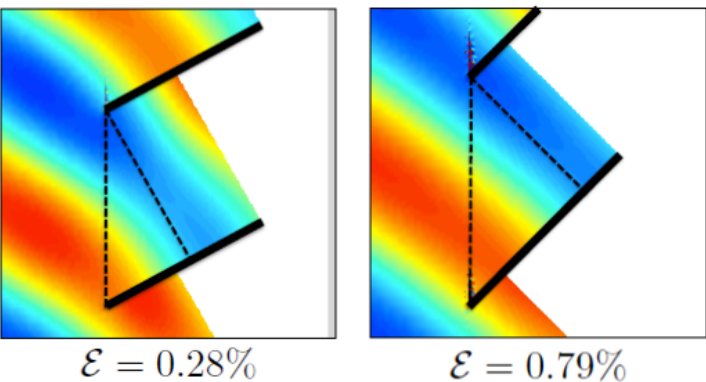

(d)

Figure 3: Transmission of an oblique incident wave at the leading-edge interface of a stator with $20^{\circ}$-staggered vanes. $M_{0}=0.3$. (a-b): compared results from Green's theorem and mode-matching technique (artifact for the former in the matching triangle due to color interpolation); (c): corresponding reflection coefficients. (d): mode-matching results for various stagger angles: $\psi=0^{\circ}$, $15^{\circ}, 30^{\circ}$ and $45^{\circ} . M_{0}=0$. Vane number $V=10$, number of lobes of the incident wave $n=7 . k h_{M}=5.57$. Instantaneous pressure field, semi-infinite plate calculations.

Sample illustrative results are displayed in Fig. 3. The test in Fig. 3-a,b,c compares the solutions obtained with the present mode-matching technique and with the application of Green's theorem described by Roger \& François. ${ }^{16}$ The stagger angle is of $20^{\circ}$ and the Mach number of 0.3. The results are in a good agreement, as confirmed by the bar-graph of the reflection coefficients in Fig. 3-c. The mode matching has the advantage of providing a uniformly valid solution whereas the Green's theorem gives access to the solution only outside the matching triangle (note that this is enough to solve the transmission problem). The figure 3-d stresses the effect of the stagger angle on the transmission of the same oblique plane wave from upstream. For instance the largest angle tested of the very-right plot obviously corresponds to a maximum transmission because the incident propagation direction is parallel to the walls. The plane-wave mode (order 0 ) is forced dominantly in the channel. In contrast the dominantly transmitted mode is the mode of order 1 in the zero-stagger case (left plot). The quality of the solution can be assessed by checking that the total wave energy is conserved. The values $\varepsilon$ mentioned below each plot indicate the relative error in the energy balance, defined as the difference between the energy of the incident wave and the sum of the energies of all scattered waves. It has been verified that the error increases with increasing Mach number and increasing stagger angle. It remains well acceptable for subsonic applications with moderate stagger angles.

\section{I.C. Effect of Vane Curvature}

\section{I.C.1. Straight-Duct Approximation}

Accurately accounting for the curvature of the inter-vane channels in the sound-transmission problem is achievable using Brambley \& Peake's multiple-scale analysis ${ }^{3}$ or the multimodal approach proposed by Félix \& Pagneux. ${ }^{4}$ These approaches involve higher complexity when compared to the present one. They could 
be implemented in a further step, keeping in mind that the present simplification is well suited for demonstrating the efficiency of the mode-matching technique. Resorting to a varying straight-duct approximation is acceptable only if the curvature effect is less important than the effect of cross-section variations. A simplified analysis in absence of flow is briefly outlined in this section to justify the approximation, ignoring the streamwise expansion of the channels. Let us consider a limited portion of a curved channel of constant height $h$, the channel center line following a circular arc of radius $R_{0}$. In the absence of flow the acoustic motion in the channel is modeled by its potential $\phi$, solution of the Helmholtz equation in polar coordinates $(\bar{r}, \theta)$. Yet if the arc is short or equivalently if its angular extent is small enough, the problem can be reduced by noting the radial coordinate $\bar{r}=R_{0}+r$ where $r$ is the deviation from the center line. With the assumption $r / R_{0} \ll 1$ and introducing the tangential coordinate $y=R_{0} \theta$, the ordinary Helmholtz operator can be recovered for the potential $\psi$ obtained by the change of variable $\phi=\mathrm{e}^{-r /\left(2 R_{0}\right)} \psi$. Furthermore the rigidity boundary condition $\partial \phi / \partial r=0$ for $r= \pm h / 2$ leads to the modified condition on $\psi$

$$
\frac{\partial \psi}{\partial r}-\frac{1}{2 R_{0}} \psi=0
$$

Straightforward derivations lead to the approximation

$$
\phi=A \mathrm{e}^{-r /\left(2 R_{0}\right)} \mathrm{e}^{\mathrm{i} \lambda_{m} R_{0} \theta}\left[\cos \left(K_{m}(r+h / 2)\right)+\frac{1}{2 R_{0} K_{m}} \sin \left(K_{m}(r+h / 2)\right)\right]
$$

for the potential of a single channel mode of order $m$, with

$$
K_{m}=\frac{m \pi}{h}, \quad \lambda_{m}=\left[k^{2}-K_{m}^{2}-\frac{1}{4 R_{0}^{2}}\right]^{1 / 2}
$$

Numerical tests not reported here have been made for modes of moderate orders and parameters representative of the stator vanes of a low-speed, small-size cooling fan, with a circular arc of $45^{\circ}$. Only retaining the cosine term in the square brackets of Eq. (6) did not induce a serious error, which means that the simple solution for the straight channel remains representative of the curved channel. A more quantitative assessment, not detailed either, has been made by expanding the modified modes on the set of cosine modes of the corresponding straight channel; the mode $\cos \left[K_{m}(r+h / 2)\right]$ has been found to be the only dominant one in the expansion, all other modes having amplitudes more than $30 \mathrm{~dB}$ lower. Obviously this analysis remains indicative and should be completed by comparing with a numerical solution. Anyway, with regards to other assumptions such as zero-thickness vanes and uniform flow in a channel cross-section, ignoring the curvature in the sense defined in this section appears as fully justified.

\section{I.C.2. Parameters of the Inter-Vane Channels}

Approximations are needed because the point $C$ of the triangle in Fig. 2-a is not exactly on the meancamber line of the vane. Conventionally we consider the mid-channel curved axis and the associated oblique straight line plotted in red in Fig. 4-a such that the line ends at the leading-edge point of the lower vane. This oblique line crosses perpendicularly the curved axis at the point marked with the red circle. It is taken as the relevant inlet cross-section of the overlapping part of the channel. According to this convention the effective length of the straightened channel is derived as

$$
L_{e}=-R_{0} \arctan \frac{\sin \psi R_{0}}{\cos \psi R_{0}+h_{M} / 2}
$$

Next taking the distance between the two crossings with the mean camber-lines of the upper and lower vanes marked with black circles, the inlet channel width follows as

$$
h_{e}=h_{M} \frac{\cos \psi R_{0}+h_{M} / 2}{\sqrt{R_{0}^{2}+\left(h_{M} / 2\right)^{2}+R_{0} h_{M} \cos \psi}}
$$

In fact the ignored thickness of the vanes makes the true channel width smaller. Furthermore because the point $C$ of the matching triangle is away from the mean-camber line the length of the segment $A C$, say $h_{0}=$ $h_{M} \cos \psi$ differs from $h_{e}$. The width $h_{0}$ and the corresponding slightly reduced length $L_{c}=\left(R_{0}-h_{M} / 2\right) \cos \psi$ 
are taken here as the relevant values instead of $h_{e}$ and $L_{e}$, because they enable an easy coupling with the mode-matching technique, on the one hand, and because they indirectly introduce some realistic thickness effect, on the other hand. Anyway the differences are only of a couple of percents, which remains acceptable with regards to other approximations made in the modeling. The channel width at any curvilinear abscissa $s$ is determined in the same way. The generated wall profiles of the straightened channels are compared in Fig. 4-b.

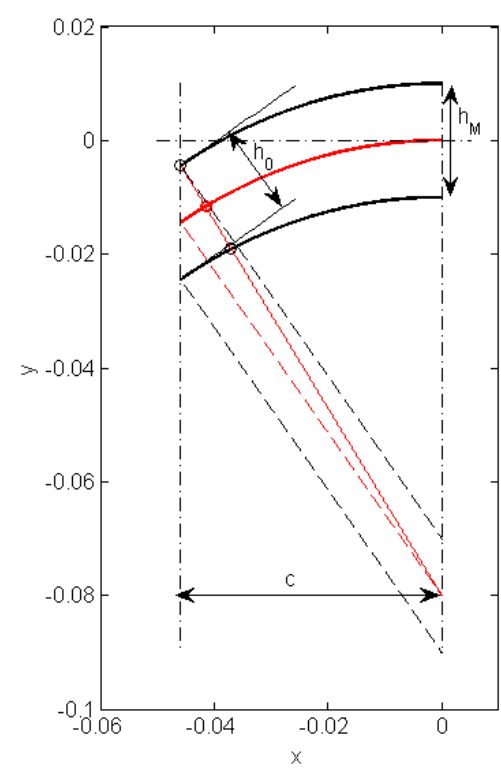

(a)

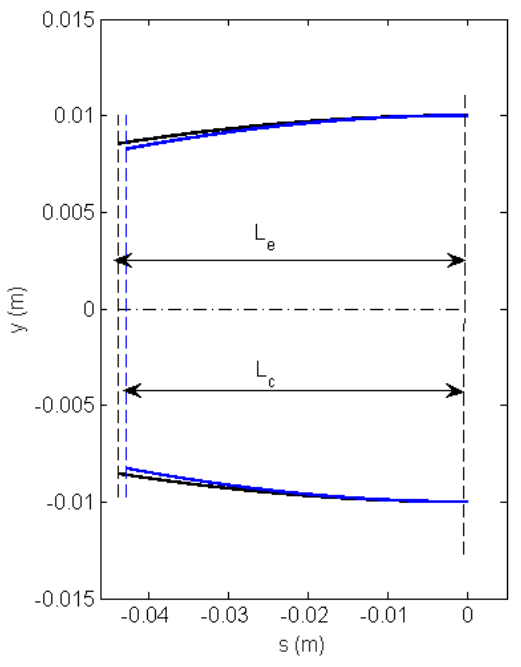

(b)

Figure 4: Representation of an inter-vane channel as an equivalent straight duct of varying cross-section, from Fig. 1-b. (a): conventions for the width and length of the overlapping part of the channel; (b): straight-duct cross-section profiles according to the two sets of parameters $\left(h_{e}, L_{e}\right)$ and $\left(h_{0}, L_{c}\right)$, in black and blue respectively. Values representative of a small-size fan for aircraft air conditioning.

\section{I.D. Slowly-Varying Duct Model}

Accepting the aforementioned simplifications, the transmission of a single mode through an inter-vane channel of slowly-varying cross-section can be described by the multiple-scale analysis. As long as the cut-on or cut-off character of the mode is not modified between the inlet and the outlet original Rienstra's formulation ${ }^{15}$ applies. If some transition occurs it must be completed as proposed by Rienstra ${ }^{14}$ and Ovenden $^{19}$ in two steps. The first step consists in calculating the transmission and reflection coefficients of the waves produced by the transition. The second step consists in regularizing the singularity at the transition. This regularization is shortly outlined here.

When a cut-on mode forced at the trailing-edge interface of the stator by equivalent trailing-edge noise sources propagates upstream and turns cut-off at some location of curvilinear coordinate $s_{t}$ it is reflected as a downstream cut-on mode of same energy and transmitted as a cut-off mode farther upstream toward the inlet. The reflection and transmission coefficients are $\mathrm{i}$ and 1 , respectively. The total potential field downstream of the transition is expressed as

$$
\phi=\frac{Q}{\sqrt{k h_{M} \sigma(\bar{X})}} \Psi(\bar{y}, \bar{X}) \exp \left(\frac{\mathrm{i}}{\varepsilon} \int_{\bar{X}_{t}}^{\bar{X}} \frac{k h_{M} M\left(\bar{X}^{\prime}\right)}{\beta^{2}\left(\bar{X}^{\prime}\right)} \mathrm{d} \bar{X}^{\prime}\right)
$$




$$
\times\left[\exp \left(\frac{-\mathrm{i}}{\varepsilon} \int_{\bar{X}_{t}}^{\bar{X}} \frac{k h_{M} \sigma\left(\bar{X}^{\prime}\right)}{\beta^{2}\left(\bar{X}^{\prime}\right)} \mathrm{d} \bar{X}^{\prime}\right)+\mathrm{i} \exp \left(\frac{\mathrm{i}}{\varepsilon} \int_{\bar{X}_{t}}^{\bar{X}} \frac{k h_{M} \sigma\left(\bar{X}^{\prime}\right)}{\beta^{2}\left(\bar{X}^{\prime}\right)} \mathrm{d} \bar{X}^{\prime}\right)\right]
$$

where $\varepsilon$ is the small parameter of the multiple-scale analysis and $\bar{X}$ a slowly varying coordinate of opposite sign to the curvilinear coordinate for compatibility with the reference paper. $\bar{y}$ is the coordinate orthogonal to $\bar{X}$. In the expression

$$
\sigma(\bar{X})=\sqrt{1-\beta^{2}(\bar{X})\left(\frac{m \pi}{k h(\bar{X})}\right)^{2}}, \quad \beta^{2}(\bar{X})=1-M^{2}(\bar{X}),
$$

where $M(\bar{X})$ is the local Mach number, negative in the present case because the incident wave propagates against the flow. $\Psi$ denotes the transverse modal function, a cosine in the present case, and $Q$ is some amplitude factor imposed by the source of the mode.

The upstream potential field of the evanescent wave reads

$$
\phi=\frac{Q \mathrm{e}^{\mathrm{i} \pi / 4}}{\sqrt{k h_{M}|\sigma(\bar{X})|}} \Psi(\bar{y}, \bar{X}) \exp \left(\frac{\mathrm{i}}{\varepsilon} \int_{\bar{X}_{t}}^{\bar{X}} \frac{k h_{M} M\left(\bar{X}^{\prime}\right)}{\beta^{2}\left(\bar{X}^{\prime}\right)} \mathrm{d} \bar{X}^{\prime}\right) \exp \left(\frac{-1}{\varepsilon} \int_{\bar{X}_{t}}^{\bar{X}} \frac{k h_{M}\left|\sigma\left(\bar{X}^{\prime}\right)\right|}{\beta^{2}\left(\bar{X}^{\prime}\right)} \mathrm{d} \bar{X}^{\prime}\right)
$$

The expressions are simplified with respect to the reference because the mean flow is assumed incompressible in the present work. Implementing the solution exhibits a singularity at the transition, as shown later in Fig. 5. Matched asymptotic expansions proposed by Ovenden ${ }^{19}$ produce a regularized solution involving the Airy function Ai, according to which the complete field has a uniformly valid expression as

$$
\begin{gathered}
\phi=\bar{Q} \Psi(\bar{y}, \bar{X})\left[-\frac{3}{2 \varepsilon} \frac{1}{\sigma^{3}} \int_{\bar{X}_{t}}^{\bar{X}} \frac{k h_{M} \sigma\left(\bar{X}^{\prime}\right)}{\beta^{2}\left(\bar{X}^{\prime}\right)} \mathrm{d} \bar{X}^{\prime}\right]^{1 / 6} \\
\times \operatorname{Ai}\left[\left(\frac{3 \mathrm{i}}{2 \varepsilon} \int_{\bar{X}_{t}}^{\bar{X}} \frac{k h_{M} \sigma\left(\bar{X}^{\prime}\right)}{\beta^{2}\left(\bar{X}^{\prime}\right)} \mathrm{d} \bar{X}^{\prime}\right)^{2 / 3}\right] \exp \left(\frac{\mathrm{i}}{\varepsilon} \int_{\bar{X}_{t}}^{\bar{X}} \frac{k h_{M} M\left(\bar{X}^{\prime}\right)}{\beta^{2}\left(\bar{X}^{\prime}\right)} \mathrm{d} \bar{X}^{\prime}\right)
\end{gathered}
$$

with $\bar{Q}=2 \sqrt{\pi} \mathrm{e}^{\mathrm{i} \pi / 4} Q / \sqrt{k h_{M}}$.

The complementary cut-off to cut-on transition can also be encountered for the same mode at the same frequency if the mode is now produced at the inlet interface instead of the outlet. This occurs:

- when sound is forced at inlet inside the channel from upstream acoustic excitation or from impingement of vortical disturbances at the leading edge;

- when partial reflection of a channel mode propagating upstream at the leading-edge interface regenerates higher-order downstream modes.

In this case the mode decays exponentially from the channel inlet to the transition point of abscissa $s_{t}$. Beyond that point the mode becomes cut-on, which means that it can be transmitted farther downstream with conservation of its energy. Now the evanescent mode between $s=0$ and $s=s_{t}$ cannot carry energy. Therefore the exponentially growing wave that is also a mathematical solution of the problem must be added in this range, which is equivalent to consider a "reflection" of the cut-off mode. The superposition of the evanescent and growing waves a priori allows energy to be transferred from the inlet to the transition point and beyond $\left(s \geq s_{t}\right)$. This is known as acoustic tunnel effect and is similar to what is described by Dowling \& Ffowcs Williams ${ }^{10}$ for oblique waves on the double interface of fluids of different densities and/or sound speeds. Because this effect possibly plays a role in sound-transmission problems through cascades when the transition takes place in the vicinity of the leading-edge interface, it has been considered in this study for completeness. Minor changes need to be made with respect to the aforementioned Rienstra's and Ovenden's analyses. First the $X$ coordinate is now defined of same sign as $s$ because the incident propagation is considered in the streamwise direction. In this positive $X$ direction the channel is diverging. The reflection and transmission coefficients are found to be i/2 and 1, respectively. This leads to the complete potential field in the cut-off part as

$$
\phi=\frac{Q \mathrm{e}^{-\mathrm{i} \pi / 4}}{\sqrt{k h_{M}|\sigma(\bar{X})|}} \Psi(\bar{y}, \bar{X}) \exp \left(\frac{\mathrm{i}}{\varepsilon} \int_{\bar{X}_{t}}^{\bar{X}} \frac{k h_{M} M\left(\bar{X}^{\prime}\right)}{\beta^{2}\left(\bar{X}^{\prime}\right)} \mathrm{d} \bar{X}^{\prime}\right)
$$




$$
\times\left[\exp \left(\frac{1}{\varepsilon} \int_{\bar{X}_{t}}^{\bar{X}} \frac{k h_{M}\left|\sigma\left(\bar{X}^{\prime}\right)\right|}{\beta^{2}\left(\bar{X}^{\prime}\right)} \mathrm{d} \bar{X}^{\prime}\right)+\frac{\mathrm{i}}{2} \exp \left(\frac{-1}{\varepsilon} \int_{\bar{X}_{t}}^{\bar{X}} \frac{k h_{M}\left|\sigma\left(\bar{X}^{\prime}\right)\right|}{\beta^{2}\left(\bar{X}^{\prime}\right)} \mathrm{d} \bar{X}^{\prime}\right)\right]
$$

and to the one downstream of the transition as

$$
\phi=\frac{Q}{\sqrt{k h_{M} \sigma(\bar{X})}} \Psi(\bar{y}, \bar{X}) \exp \left(\frac{\mathrm{i}}{\varepsilon} \int_{\bar{X}_{t}}^{\bar{X}} \frac{k h_{M} M\left(\bar{X}^{\prime}\right)}{\beta^{2}\left(\bar{X}^{\prime}\right)} \mathrm{d} \bar{X}^{\prime}\right) \exp \left(\frac{-1}{\varepsilon} \int_{\bar{X}_{t}}^{\bar{X}} \frac{k h_{M}\left|\sigma\left(\bar{X}^{\prime}\right)\right|}{\beta^{2}\left(\bar{X}^{\prime}\right)} \mathrm{d} \bar{X}^{\prime}\right)
$$

Though the developments are not detailed here the associated uniformly valid solution is found as

$$
\begin{gathered}
\phi=\bar{Q} \Psi(\bar{y}, \bar{X})\left[-\frac{3}{2 \varepsilon} \frac{1}{\sigma^{3}} \int_{\bar{X}_{t}}^{\bar{X}} \frac{k h_{M} \sigma\left(\bar{X}^{\prime}\right)}{\beta^{2}\left(\bar{X}^{\prime}\right)} \mathrm{d} \bar{X}^{\prime}\right]^{1 / 6} \\
\times \mathrm{F}_{\mathrm{AB}}\left[-\left(\frac{3 \mathrm{i}}{2 \varepsilon} \int_{\bar{X}_{t}}^{\bar{X}} \frac{k h_{M} \sigma\left(\bar{X}^{\prime}\right)}{\beta^{2}\left(\bar{X}^{\prime}\right)} \mathrm{d} \bar{X}^{\prime}\right)^{2 / 3}\right] \exp \left(\frac{\mathrm{i}}{\varepsilon} \int_{\bar{X}_{t}}^{\bar{X}} \frac{k h_{M} M\left(\bar{X}^{\prime}\right)}{\beta^{2}\left(\bar{X}^{\prime}\right)} \mathrm{d} \bar{X}^{\prime}\right)
\end{gathered}
$$

with $\mathrm{F}_{\mathrm{AB}}=\mathrm{Ai}-\mathrm{i} \mathrm{Bi}$. It now also involves the Airy function of the second kind Bi.

The cut-on to cut-off transition of an upstream-propagating mode in an inter-vane channel is illustrated in Fig. 5. The flow is from left to right and the incident mode of order $m=3$ propagates against the flow from the right-hand side. At the considered Helmholtz number $k h_{M}=9.25$ and for the considered stator geometry the mode is reversed because of the transition around the curvilinear coordinate $s_{t}=0.018$. A standing-wave pattern is formed by the superimposed incident and reflected modes. The pressure-amplitude profile is plotted as a function of the curvilinear coordinate along the curved axis of the channel in Fig. 5a, where the initial solution without regularization (black) is compared with the uniformly valid solution (red). The singular peak in the former points the location of the transition. A corresponding regularized instantaneous pressure map is shown in Fig. 5 -b, featuring the three nodal lines expected for the mode $m=3$. A rapid amplitude drop is observed in the cut-off part close to the inlet section, so that this mode will not be transmitted upstream of the leading-edge. However other modes for which transition occurs closer to the inlet could have a sufficient residual amplitude to contribute to the matching at the leading-edge. At the trailing-edge interface (outlet) the mode must be combined with its reflection when writing the matching equations. A node of the standing wave is also observed around the cross-section of abscissa $s=0.04$.
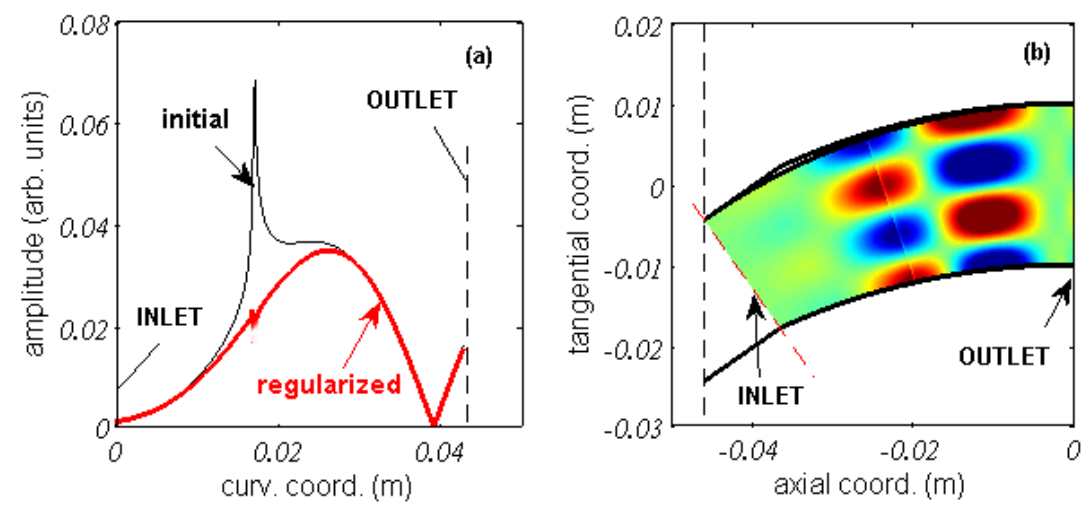

Figure 5: Example of standing-wave pattern due to cut-on to cut-off transition for the mode $m=3$ at $k h_{M}=9.25$ and $M_{0}=0.35$, in a curved channel of curvature radius $4 h_{M}$ (stagger angle at leading edge $35^{\circ}$ ). (a): pressureamplitude profile along the curved channel axis, according to initial Rienstra's solution (black) and to regularized Ovenden's solution (red). (b): instantaneous pressure map. 


\section{I.E. Coupled Bent-and-Staggered Vane Model}

The complete coupling with different stagger angles at leading and trailing edges finally involves three distinct models, namely:

- the classical mode-matching at the trailing-edge interface, as in the reference; ${ }^{18}$

- the inlet mode-matching achieved by the extension of the section I.B;

- the multiple-scale analysis for the channel transmission, including a treatment of the cut-on to cut-off and cut-off to cut-on transitions.

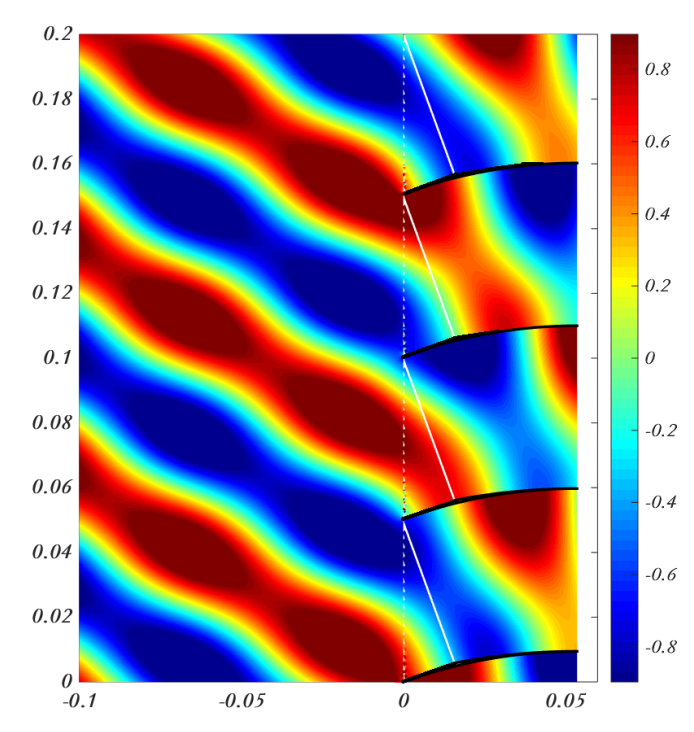

(a)
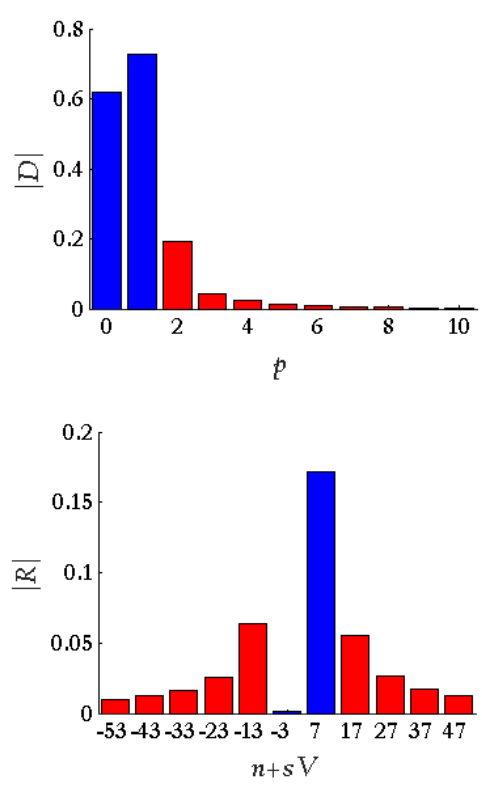

(b)

Figure 6: Test of coupled mode-matching at inlet and sound transmission through curved inter-vane channels. (a): instantaneous pressure field. No reflection at the trailing-egde interface. Incident oblique wave from upstream.(b): modal transmission (D) and reflection (R) coefficients. Blue (red) bars stand for cut-on (cut-off) modes. Incident mode $n=7, V=10, \psi=20^{\circ}, M_{0}=0.1$, $k h_{M}=5.57$.

A first example of coupling of the mode-matching procedure at the inlet of a staggered cascade with the sound-transmission model in a series of curved channels is shown in Fig. 6. For this preliminary test the channel exit interface is not considered; this is equivalent to apply a non-reflective condition at the trailing-edge. The matching triangles are featured by the thin dashed white lines. The picture is a zoom of the complete unwrapped field for an incident wave with 7 lobes on a stator of 10 vanes. It confirms the continuity of the pressure field. The wiggled wavefronts result from the superposition of the dominant reflected mode of order 7 on the incident mode. No special feature related to mode transitions is seen in this case. It must be noted that the $D$ coefficients shown in the upper right plot are defined on the side BC of the matching triangle. They are exactly the required input for the slowly-varying duct formulation of the inter-vane channel transmission.

\section{Approximate Three-Dimensional Formulations}

\section{II.A. The Zero-Stagger Flat-Plate Configuration}

\section{II.A.1. Problem Statement}

As shown by Roger et al. ${ }^{18}$ the trailing-edge noise sources of an isolated flat-plate airfoil or half-plane at some spanwise location can be reproduced by approaching an equivalent lift dipole very close to the edge. 
The strength of this edge-dipole depends on the local statistical properties of the hydrodynamic wall-pressure statistics in the plate boundary-layer but not on the presence of additional surfaces. Once it is determined its direct radiated field can be used to calculate the scattering by any body of arbitrary geometry. In the present case the expansion is obtained as a sum of Fourier-Bessel modes according to the section II.A.3 below. Then the trailing-edge noise formulation reduces to a sound-diffraction problem by an annular array of bifurcated waveguides. As a first insight, an exact three-dimensional mode-matching solution can be derived for flatplate vanes aligned with the duct axis, thus included in meridian planes as shown in Fig. 7 (zero stagger). The developments are detailed in a companion paper, ${ }^{2}$ dealing with rotor-stator wake-impingement noise. The method is just outlined here. As in the two-dimensional case it relies on two steps.

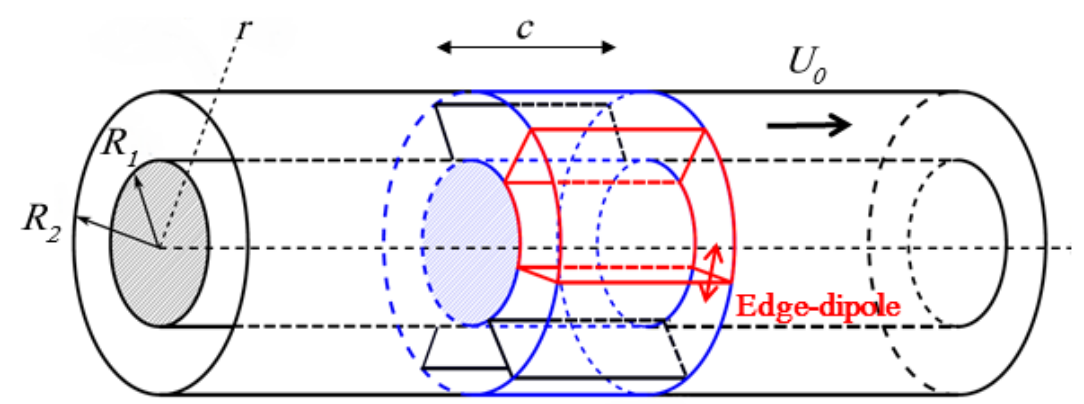

Figure 7: The annular cascade of zero-stagger plates.

1. The scattered potentials are expressed in various subdomains (annular duct segments upstream and downstream of the stator, inter-vane channels) as sums of modes, each of which satisfies the convected Helmholtz equation in cylindrical coordinates and the local rigid-wall boundary conditions. In the annular parts the radial shape functions of the modes are the classical linear combinations of Bessel functions of the first and second kinds. Inside the inter-vane channels the orders of the Bessel functions are $m V / 2$ if $V$ is the number of vanes because of the angular boundaries of the channels. ${ }^{9}$ The amplitudes of all modes are the unknowns of the problem.

2. The leading-edge and trailing-edge cross-sections of the stator are considered as interfaces at which the fluctuating pressure and axial velocity must be continuous. Thanks to suitable mathematical projections the resulting matching equations lead to linear systems that are solved by matrix inversion.

\section{II.A.2. Modal Expansion of the Trailing-Edge Dipole}

Considering a point edge-dipole of strength $F_{t}$ is enough to illustrate the method. Indeed trailing-edge noise sources distributed along a vane are known to have a very small spanwise correlation length, negligible when compared to the acoustic wavelengths of interest. Therefore they can be replaced by an equivalent distribution of uncorrelated discrete dipoles along a (radial) line very close to the trailing edge.

In the present case the lift dipole only has a tangential component because its axis is normal to the surface of the vane. Its direct field in the duct, assuming a uniform axial flow, is simply expressed by forming the product of the dipole strength with the azimuthal gradient of the Green's function for the convected Helmholtz equation. The Green's function reads ${ }^{8}$

$$
\begin{gathered}
G_{\omega}\left(\mathbf{r} / \mathbf{r}_{0}\right)=\frac{i}{4 \pi} \sum_{m=-\infty}^{\infty} \sum_{j=0}^{\infty} \frac{P_{n j}(r) P_{n j}\left(r_{0}\right)}{\sqrt{k^{2}-\beta^{2} K_{n j}^{2}}} e^{i m\left(\theta-\theta_{0}\right)} \ldots \\
\ldots e^{-i \frac{M_{0} k}{\beta^{2}}\left(z-z_{0}\right)} e^{i \frac{\left|z-z_{0}\right|}{\beta^{2}} \sqrt{k^{2}-\beta^{2} K_{n j}^{2}}}
\end{gathered}
$$


for a dipole located at $\mathbf{r}_{0}=\left(r_{0}, \theta_{0}, z_{0}\right) . P_{n j}$ with $n=|m|$ denotes the normalized radial functions, combinations of Bessel functions, such that

$$
\int_{R_{1}}^{R_{2}} r P_{n j}(r) P_{n i}(r) d r=\delta_{i j}
$$

and $K_{n j}$ the radial wavenumbers.

Therefore the dipole field is obtained as

$$
p_{\omega}(\mathbf{r})=\sum_{m=-\infty}^{\infty} \sum_{j=0}^{\infty} A_{m j}^{ \pm} P_{n j}(r) \mathrm{e}^{\mathrm{i} m \theta} \mathrm{e}^{\mathrm{i} k_{n j}^{ \pm} z}
$$

with

$$
A_{m j}^{ \pm}=\frac{m F_{t}}{4 \pi r_{0}} \frac{P_{n j}\left(r_{0}\right) \mathrm{e}^{-\mathrm{i} m \theta_{0}} \mathrm{e}^{-\mathrm{i} k_{n j}^{ \pm} z_{0}}}{\sqrt{k^{2}-\beta^{2} K_{n j}^{2}}}
$$

\section{II.A.3. Sample Results}

A typical instantaneous pressure field around an annular cascade of zero-stagger plates, as produced by the mode-matching for a single point source at the trailing edge of a reference vane, is plotted in Fig. 8-a. The parameters are representative of a small-size fan used in aeronautical air conditioning systems. The number of vanes is $V=23$, the tip radius is $R_{2}=85 \mathrm{~mm}$ and the hub-to-tip ratio is 0.55 . The axial flow speed is of $36 \mathrm{~m} / \mathrm{s}$. The computations are performed at $9 \mathrm{kHz}$; at this frequency the vane-to-vane distance at tip is close to half the acoustic wavelength. The point lift-dipole is placed at the trailing-edge of the center vane at mid span (50\% of annulus height). Three full unwrapped cylindrical cuts of the three-dimensional sound field are displayed, at $85 \%, 50 \%$ and $15 \%$, respectively. The different pitch-to-wavelength ratios and solidities are clearly identified. The source is at the coordinates $(x=c ; y=0)$ in the center map. Opposite phases are observed in the upper and lower halves of each map, as expected, but very different wavefront patterns are formed, which illustrates the three-dimensionality of the radiation. Furthermore overall phase oppositions are identified at the different radii, which is explained by the fact that the height $\left(R_{2}-R_{1}\right)$ is of the same order of magnitude as the wavelength. Another source location close to the outer radius $(95 \%)$ is tested in Fig. 8-b for comparison with a two-dimensional prediction made for a different chord but same frequency and vane-to-vane distance. The unwrapped cut is made at the source radius. Similar interferences are qualitatively observed, which suggests that the structure of the scattered field is primarily a matter of compared wavelength and vane-to-vane distance. This also confirms the cross-consistency of the two-dimensional and three-dimensional models.

It is worth noting that as already pointed for the two-dimensional implementation, ${ }^{16}$ the zero-stagger model is unable to make the plane-wave mode observable when it should be generated by virtue of the modal scattering. Indeed all secondary sources corresponding to the diffraction are equivalent to in-phase dipoles normal to the vanes. For this reason the field upstream of the cascade might be unrealistic when the plane-wave mode contribution is significant, typically at low frequencies for which the modal density of the propagating field is low. This motivates further extensions to account for the vane curvature, as shortly introduced in the next section.

\section{II.B. Towards 3D Modeling of Staggered Vanes}

The present ongoing work is also aimed at contributing by accounting for a non-zero stagger at leading edge. This is believed achievable by generalizing the approach described in section I, at the price of additional simplifications because the inter-vane channels are now twisted. A three-dimensional version of the multiplescale analysis is required, based on the same straight-duct approximation.

It is argued in the present study that the curvature of the inter-vane channels remains moderate. The vanes are assumed thin enough to be reduced to their mean-camber surface. The tangent surface to the mean-camber surface at leading edge initially follows a pure helical path of constant angle; that angle is equal to the stagger angle at leading edge, defined with respect to the duct axis. This ensures that the unwrapped representation of the stator leading-edge region would fit with an array of parallel staggered plates as shown in Fig. 1-b. Furthermore it is assumed that the non-overlap region (between leading edge 

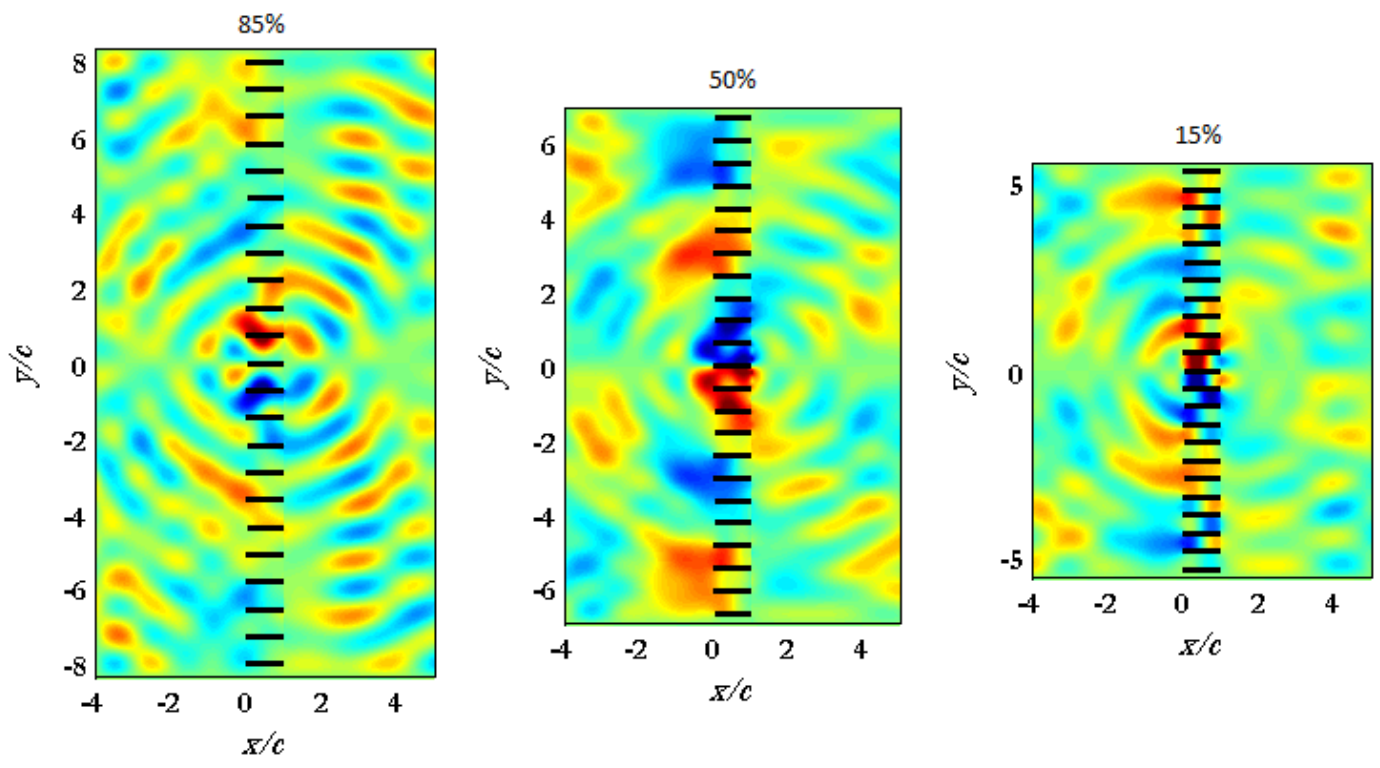

(a)
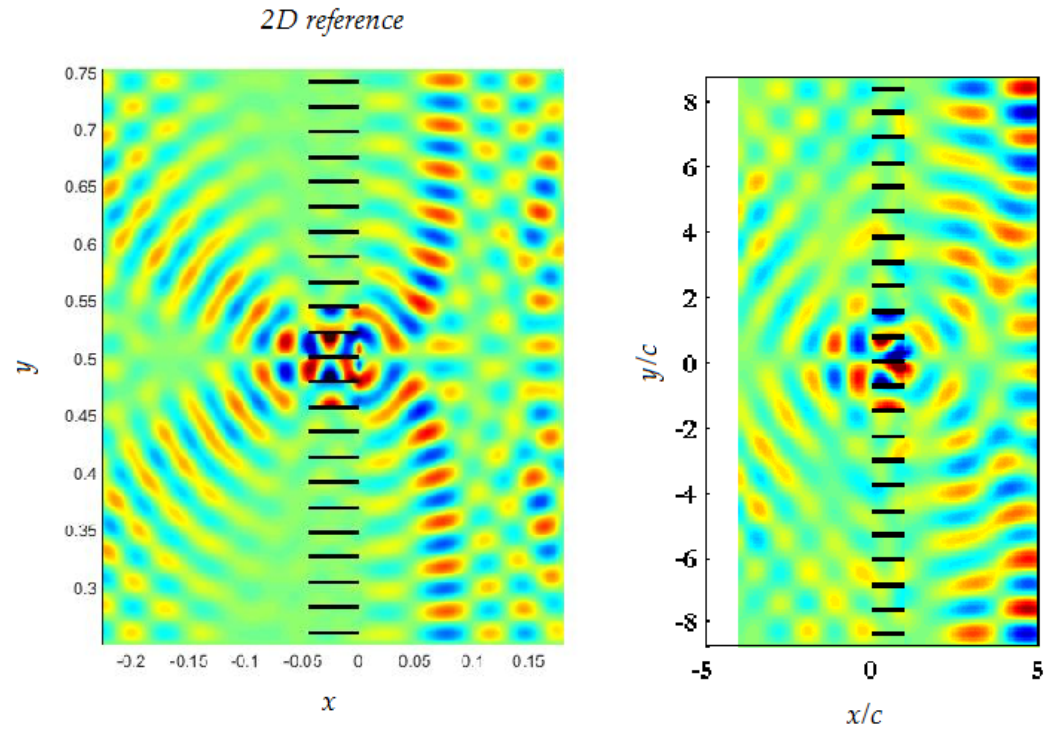

(b)

Figure 8: Typical radiation patterns of a trailing-edge noise source at the center vane of an annular cascade. $R_{1} / R_{2}=0.55, k R_{2}=14.1$, axial Mach number 0.1 . $c=3 \mathrm{~cm}$. (a): unwrapped annular cuts of the instantaneous pressure field at various percentages of the total duct height. (b): other test with the same parameters but with the source and the annular cut at $95 \%$ of duct height (right), compared to a similar two-dimensional test case ${ }^{18}$ (left). Flow from left to right. 
and point $\mathrm{C}$ in Fig. 1-b) extends only over a reasonably small distance from the leading edge, so that it can be described quite well by reducing the mean-camber surface to its tangent surface. The approximation makes sense only if the deviation remains much smaller than other characteristic lengths such as the width of the inter-vane channel.

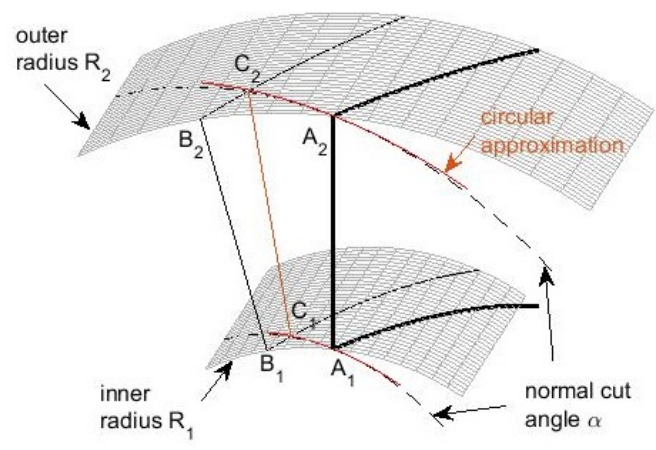

(a)

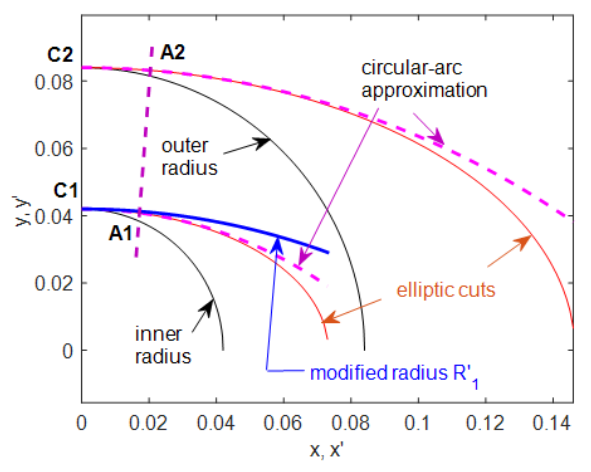

(b)

Figure 9: Geometrical simplifications at the inlet of an annular staggered array of OGV. (a): three-dimensional view of the inlet of an inter-vane channel. (b): inlet cross-section normal to the walls. (a): definition of the matching volume and of the equivalent circular arcs along an oblique cut normal to vane leading edge. (b): definition of approximate circular arcs.

On the three-dimensional sketch in Fig. 9-a the non-overlap region is defined as the region delimited by the stator front face (the duct cross-section containing the leading edges) and the oblique plane normal to the mean-camber surface at leading edge. It will be referred to as the matching volume of an inter-vane channel. This volume is defined by the two sets of points A1-B1-C1 and A2-B2-C2. It is bounded radially by the hub and tip cylinders of radii $R_{1}$ and $R_{2}$. For each inter-vane channel, the aforementioned oblique plane generates a cut of the annular duct walls. At the hub and tip radii this cut features ellipses on the inner and outer cylinders. But over the limited angular extent of a channel $2 \pi / V$ the elliptic arcs A1-C1 and A2-C2 in Fig. 9-a can be approximated by the circular arcs of local curvature radii $R_{1} / \cos ^{2} \psi$ and $R_{2} / \cos ^{2} \psi$, respectively. These local arcs do not have a common curvature center anymore. In order to recover a single center for both arcs and the same channel height $h=R_{2}-R_{1}$ another approximation is made for the inner arc by introducing the modified radius $R_{1}^{\prime}=R_{1}+R_{2} \tan ^{2} \psi$ in place of $R_{1} / \cos ^{2} \psi$. As shown in Fig. 9-b the approximation is reasonable for large vane numbers. Along the oblique cut the tangential extent of the inter-vane channel is now $2 \pi \cos \psi R_{2} / V$ at the outer radius, which is equivalent to an aperture angle $(2 \pi V) / \cos ^{3} \psi$, thus to a virtual vane number $V^{\prime}=V / \cos ^{3} \psi$ generally not an integer. From the standpoint of sound transmission inside a single channel, the non-zero stagger appears as equivalent to an increased mean radius at constant height (equivalently an increased hub-to-tip ratio) and to an increased vane number. The areas of the front section of a channel in the duct cross-section and of the channel cross-section in the normal cutting plane follow as

$$
\frac{\pi h}{V}\left(R_{1}+R_{2}\right) ; \quad \frac{\pi h}{V} \cos ^{3} \psi\left[R_{1}+\frac{1+\sin ^{2} \psi}{\cos ^{2} \psi} R_{2}\right] .
$$

In the limit $R_{1} \rightarrow R_{2}$ the second expression becomes $\cos \psi$ times the first one, similar to the twodimensional unwrapped case. This ensures the conservation of mass or flow rate for an incompressible fluid. But because of the approximations the conservation is not satisfied in the general case; the associated inaccuracy can be quantified by the flow-rate ratio

$$
\frac{R_{1} \cos ^{2} \psi+R_{2}\left(1+\sin ^{2} \psi\right)}{R_{1}+R_{2}}
$$

For moderate stagger angles and large hub-to-tip ratios such as in the configuration of Fig. 9-a and for subsonic rotor-stator stages, assimilating the ratio to 1 is acceptable. Finally the oblique uniform flow speed 
$U=U_{a} / \cos \psi$ will be considered as the approximate value at the channel entrance if $U_{a}$ is the axial flow speed.

Describing sound propagation inside a single inter-vane channel once the matching at inlet is solved is a key step of the proposed approach. It is again assumed that the channel curvature can be ignored as of secondary importance and that the dominant effect is rather the continuous variation of the channel cross-section normal to its curved centerline. The inter-vane channel is virtually unbent so that it can be modeled using the multiple-scale analysis developed by Rienstra ${ }^{14}$ and Ovenden. ${ }^{19}$ The consequence is that the parameters of any channel mode will be allowed to vary continuously from leading edge to trailing edge and vice versa, the emphasis being on the effects of the upstream-to-downstream expansion of the channel cross-section. In particular the same cut-on to cut-off and cut-off to cut-on transitions as for the two-dimensional model of section I are expected. It is worth noting that the complementary mode matching at the trailing-edge interface can be formulated exactly in cylindrical coordinates as in section II.A, in the present configuration of zero stagger at the trailing edge.

The whole set of approximations made with the oblique cuts at the inlet of the stator can be repeated at any downstream location along a curved channel. Let $s$ be the coordinate along the rectilinear channel axis and now $R_{1}^{\prime}(s), R_{2}^{\prime}(s)$ be the local hub and tip radii. The local angular aperture of the channel is defined by the virtual vane number $V^{\prime}(s)=V / \cos ^{3} \psi(s)$. The generic expression of the radial function of a channel mode reads

$$
J_{\mu(s)}\left(K_{m j}(s) r\right)+A_{m j}(s) J_{\mu(s)}\left(K_{m j}(s) r\right)
$$

with $\mu(s)=m V^{\prime}(s) / 2$ and the associated azimuthal function is $\cos [\mu(s) \theta]$, the polar coordinates being defined in the plane normal to the channel center line.

This closes the set of mathematical tools required for implementing the mode-matching technique. The complete procedure that reproduces the chaining of involved physical mechanisms is as follows.

- Each mode of amplitude defined by Eq. (15) is first scattered at the trailing-edge interface using the model of section II.A (for incident upstream propagation). For this step a three-dimensional Kutta condition must be implemented, as described by François et al. ${ }^{21}$

- The transmitted waves propagate farther upstream according to a three-dimensional declination of the multiple-scale model of section I.C; the implementation of this part is presently in progress.

- The next step consists in performing another mode matching at inlet in which the matching volume of Fig. 9-a is the equivalent of the matching triangle of Fig. 2. This is the most difficult point to solve mathematically. Indeed the definition of the duct modes upstream of the stator has to be re-addressed because the actual mean flow is swirling there, making the ordinary Helmholtz equation invalid. In view of the mathematical complexity that an exact statement of the effects of swirl would introduce (see for instance Posson \& Peake ${ }^{13}$ ), additional simplifications could be justified for archtectures in which the swirl extends only over a limited axial distance, with a small swirling Mach number.

\section{Conclusion}

The new formulation proposed in the paper is aimed at solving sound-generation and sound-transmission problems in axial-flow turbomachinery stages by making an acceptable compromise between analytical tractability and the preservation of an as-realistic-as-possible geometry. In particular the proposed method avoids resorting to a strip-theory approach. It has been described for a stationary row of outlet guide vanes and only for the transmission of an acoustic wave but it can be transposed to a rotating blade row by a change of reference frame and/or to the excitation by hydrodynamic disturbances. Key steps of the cascade scattering mechanism are addressed, the complete chaining of which, still in progress, is out of the purely methodological scope of the paper. This allows pointing out separately physical effects involved in turbomachinery noise.

The proposed method makes extensive use of the mode-matching technique: the field is expanded as sets of normal modes in each sub-domain (inter-vane channels and inlet and outlet annuli) that are matched by imposing the continuity of the acoustic pressure and streamwise velocity. In particular this allows introducing inter-vane channels of varying cross-section, therefore taking into account the vane curvature, which is an improvement with respect to alternative analytical approaches. The effect of the stagger angle at the inlet of a stator and the effect of vane curvature have been first analyzed separately in a two-dimensional context. A preliminary coupling also enabled to simulate the inlet transmission of an oblique wave into a stator with a realistic vane shape, ensuring a continuous re-orientation of the mean flow from an incident oblique 
direction to the axial direction at outlet (swirl recovery). The divergence of the inter-vane channels is shown to be responsible for two effects that are accounted for with a multiple-scale analysis. Firstly upstreampropagating modes produced by trailing-edge noise sources can be reversed because of cut-on to cut-off transition. Secondly, the complementary cut-off to cut-on transition can induce an acoustic tunnel effect by which sound energy is pumped from upstream if the involved modes are cut-off at channel inlet but cut-on farther downstream.

In a second step the mode-matching technique has also been implemented in a three-dimensional context to model the sound transmission through a cascade of zero-stagger vanes. According to the edge-dipole theory previously developed in $2 \mathrm{D}$, this model can be used to predict cascade trailing-edge noise at the price of a modal expansion of the source terms. First tests illustrating the radiation of a point trailing-edge noise source in the annular duct have been presented, confirming the consistency of the approach.

Finally the extension of the 3D model to account for staggered and curved vanes has been discussed and preliminary simplifications and guidelines have been established. This extension is presently in progress.

\section{Acknowledgements}

The present developments have been partially made in the EC-funded project ACP2-GA-2012-314066 IDEALVENT (Integrated Design of Optimal Ventilation Systems for Low Cabin and Ramp Noise) and in the research program of the industrial Chair ADOPSYS co-funded by SAFRAN-Snecma and the French ANR. The authors are grateful to S. Bouley for his contribution to the 3D trailing-edge noise modeling.

\section{References}

${ }^{1}$ S. Bouley, B. François, M. Roger, H. Posson \& S. Moreau, On a mode-matching technique for sound generation and transmission in a linear cascade of outlet guide vanes, 21 $1^{\text {st }}$ AIAA/CEAS Aeroacoustics Conference, Aviation 2015, Dallas, paper 2015-2825, 2015.

${ }^{2}$ S. Bouley, B. François \& M. Roger, On a mode-matching technique for sound generation and transmission in a threedimensional annular cascade of outlet guide vanes, 22d AIAA/CEAS Aeroacoustics Conference, Lyon, 2016

${ }^{3}$ E. Brambley \& N. Peake, Sound Transmission in Strongly-Curved Slowly-Varying Cylindrical and Annular Lined Ducts With Flow, AIAA paper 2006-2582, 2006.

${ }^{4}$ S. Félix \& V. Pagneux, Sound propagation in rigid bends: a multimodal approach, J. Acoust. Soc. Amer., vol. 110(3-1), pp. 1329-1337, 2001.

${ }^{5}$ S.A.L. Glegg, The response of a swept blade row to a three-dimensional gust, J. Sound Vib., vol. 227(1), pp.29-64, 1999.

${ }^{6}$ D.B. Hanson, Theory of Broadband Noise for Rotor and Stator Cascade with Inhomogeneous Inflow Turbulence Including Effects of Lean and Sweep, NASA CR-210762, 2001

${ }^{7}$ S.A.L. Glegg \& C. Jochault, Broadband self-noise from a ducted fan, $3^{\text {rd }}$ AIAA/CEAS Aeroacoustics Conference, Atlanta, 1997.

${ }^{8}$ M.E. Goldstein, Aeroacoustics, McGraw-Hill, NY, 1976.

${ }^{9} \mathrm{~J}$. Ingenito \& M. Roger, Analytical modeling of sound transmission through the passage of a centrifugal compressor, $13^{\text {th }}$ AIAA/CEAS Aeroacoustics Conference, paper 2007-3704, Rome, 2007.

${ }^{10}$ A.P. Dowling \& J.E. Ffowcs Williams, Sound and sources of sound, Horwood, 1983

${ }^{11}$ R. Mittra \& S.W. Lee, Analytical Techniques in the Theory of Guided Waves, McMillan, NY, 1971.

${ }^{12} \mathrm{H}$. Posson, M. Roger \& S. Moreau, On a uniformly valid analytical rectilinear cascade response function, J. Fluid Mech., vol. 663, pp. 22-52, 2010 .

${ }^{13}$ H. Posson \& N. Peake, Acoustic analogy in swirling mean flow applied to predict rotor trailing-edge noise, $18^{t h}$ AIAA/CEAS Aeroacoustics Conference, paper 2012-2267, Colorado Springs, 2012.

${ }^{14} \mathrm{~S}$. W. Rienstra, Sound propagation in slowly varying lined flow ducts of arbitrary cross-section, J. Fluid Mech. 495, pp. 157-1736, 2003.

${ }^{15} \mathrm{~S}$. W. Rienstra, Sound transmission in slowly varying circular and annular lined ducts with flow, J. Fluid Mech. 380, pp. 279-296, 1999.

${ }^{16}$ M. Roger \& B. François, Combined Analytical Models for Sound Generation and Transmission in Cambered Axial-Flow Outlet Guide Vanes, ISROMAC16, paper 58, Honolulu HA, 2016.

${ }^{17}$ J. de Laborderie, V. Blandeau, T. Nodé-Langlois \& S. Moreau, Extension of a fan tonal noise cascade model for camber effects, AIAA Journal, vol. 53(4), 2015.

${ }^{18}$ M. Roger, B. François \& S. Moreau, Towards Cascade Trailing-Edge Noise Modeling Using a Mode-Matching Technique, 20 ${ }^{\text {th }}$ AIAA/CEAS Aeroacoustics Conference, AIAA paper 2015-2541, Dallas, 2015.

${ }^{19} \mathrm{~N}$. Ovenden, A uniformly valid multiple scales solution for cut-on cut-off transition of sound in flow ducts, J. Sound $\mathcal{E}^{\circ}$ Vib. 286, pp. 403-416, 2005.

${ }^{20}$ E.A.N. Whitehead, The Theory of Parallel-Plate Media for Microwave Lenses, Proc. IEE London, 98 (part III), pp. 133$140,1951$. 
${ }^{21}$ B. François, S. Bouley \& M. Roger, On the Kutta condition for sound transmission in an annular cascade of vanes, ISROMAC16, paper 58, Honolulu HA, 2016. 